sondern war ihnen untertan ... Die weltliche Gewalt, selbst geistlich gefärbt, besaß die volle Überlegenheit und konnte, ohne daß3 Widerspruch laut wurde, sogar tief in das innere Leben der Kirche eindringen." Man vermift hier eine Erinnerung an die rechtliche Stütze dieser Kirchherrschaft unserer Kaiser, an die Erstreckung des eigenkirchlichen Gedankens wie auf niedere Kirchen so auf Abteien, Bistümer und schlieflich gar über das Papsttum, die eben um ihrer Sättigung willen den Angriff des nach Autonomie verlangenden römisch-rechtlichen Universalismus möglich und erfolgreich machte. Eben dieser Rechtsgedanke der Eigenkirche lebte aber wieder auf im landesherrlichen Patronat des späteren Mittelalters, nur daß er jetzt räumlich ausgedehnt und in seiner Verbindung mit der Territorialhoheit publizistisch umgeprägt worden war: in neuer Gestalt, aber in alter Kraft stand er gleichsam Pate bei den Ansätzen von Landeskircben, um zu seinem Teil die Vogtgewalt des Landesherren zu verstärken.

Königsberg i. Pr.

A. Werminghoff.

\title{
Adolf Franz, Das Rituale des Bischofs Heinrich I. von
} Breslau. Freiburg i. Br., Herder 1912. XI, $91 \mathrm{~S}$.

Es bedarf wohl kaum einer Begründung, wenn die Veröffentlichung einer Ritualienhandschrift in diesem Organ eine Anzeige findet. Denn wer sich auch nur mit den eherechtsgeschichtlichen Arbeiten von Emil Friedberg und Rudolf Sohm beschäftigt hat, weis zur Genüge, welch bedeutender rechtsgeschichtlicher Gehalt dieser zunächst liturgiegeschichtlich bemerkenswerten Quellengattung zukommt. Freilich die soeben erwähnten Autoren befanden sich keineswegs in einer günstigen Lage, als sie für die Zwecke ihrer Untersuchung Ritualien benötigten. Sie waren ja im wesentlichen auf Martènes Werk „De antiquis ecclesiae ritibus" (Antwerpen 1763/1764, die Antwerpener Ausgaben sind bei weitem besser als andere) angewiesen. In dieser gewifs mit Riesenfleiß hergestellten und geradezu unschätzbaren Sammlung fanden sie nun ein Material vor, das sich in der Hauptsache nun doch auf französische Territorien beschränkte. Dazu kam noch, der Oratorianer bot und bietet die liturgischen Texte in einer Datierung, die den Benutzer auf Schritt und Tritt in Verlegenheit setzt. Allerdings es ist verständlich, wenn die Nichtliturgiker Friedberg und Sohm letzterem Punkte keine sonderliche Aufmerksamkeit schenkten.

Der erste Rechtshistoriker, der sich der Erforschung der Ritualien man spricht im gleichen Sinne auch von Pastorale, Obsequiale, Sacerdotale, Agende, Manualia oder Liber Benedictionum - mit ungleich gröferem liturgischen Feingefühl widmete, ist Joseph Freisen. Seine zahlreichen Arbeiten sind sicherlich Richtung weisend, wenn sie auch, wie mir scheint, in den Kreisen der Rechtshistoriker längst noch nicht die gebührende Aufmerksamkeit gefunden haben. Freisen eignet einmal 
das Verdienst, er bereicherte unsere Kenntnis von den Diözesanritualien durch eine Reihe trefflicher Editionen, dann aber hat er auch unseren Blick für den rechtsgeschichtlichen Quellenwert dieser liturgischen Formulare bedeutend geschärft (vgl. besonders dessen Abhandlung "Die katholischen Ritualbücher der nordischen Kirche und ibre Bedeutung für die germanische Rechtsgeschichte" in Deutschrechtliche Beiträge, hrsg. von Beyerle, III, Heidelberg 1909, S. $137 \mathrm{ff}$.).

Den Liturgiehistoriker Adolf Franz, zweifelsohne eine der ersten liturgischen Autoritäten der Gegenwart, leitete vor allem das Interesse am historischen Kultleben, wenn auch er und schlieflich früher als alle anderen (Schönfelder, Stapper) der Ritualienforschung sich zuwandte. Sowohl in seiner "Messe im deutschen Mittelalter" (Freiburg i. Br. 1902) wie auch in seinem zweibändigen Werk "Die kirchlichen Benediktionen im Mittelalter" (Freiburg i. Br. 1909) bat uns dieser Gelehrte in geradezu verschwenderischer Fülle Textproben und Quellenstellen aus zumeist unedierten oder doch schwer zugänglichen Ritualien mitgeteilt. In seiner durchaus vorbildlichen Ausgabe des Rituale von St. Florian (Freiburg i. Br. 1904) nahmen diese mit großer Energie und mit entsagungsvoller Hingabe durchgeführten Arbeiten monographische Gestalt an. Es braucht bei einem so universal veranlagten und tief schürfenden Forscher, wie es Franz ist, kaum bemerkt zu werden: er ging bei seinen gewifs vorwiegend liturgiegeschichtlich gerichteten Jntersuchungea rechtsgeschichtlichen Problemen keinesfalls aus dem Wege. ${ }^{1}$ ) Ja man dürfte unserer Behauptung sicherlich zustimmen, wenn wir sagen, die kirchenrechtsgeschichtliche Forschung der letzten Jahre kann nur auf wenige Werke zurückblicken, die ihr so viel Auregung geben wie ein Buch, das doch schlieflich einem Nachbargebiet angehört: und das sind die bereits erwähnten Untersuchungen von Franz über die mittelalterlichen kirchlichen Segnungen.

Die uns heute von dem nämlichen Autor vorgelegte Veröffentlichung enthält das Rituale des Bischofs Heinrich I. von Breslau (1302 bis 1319). Sie bietet einmal den Text der Handschrift und dazu noch Erläuterungen. Dieses Rituale ist, ohne dafo sich über seinen Verfasser näheres sagen ließse, unter dem Pontifikat dieses Kirchenfürsten entstanden. Es galt zunächst für den Dom, dann aber und unter den notwendigen Abänderungen auch für die Kirchen der Diözese. Der spezifisch liturgischen Bedeutung der erstmalig veröffentlichten und inhaltreichen Handschrift kann an dieser Stelle natürlich nicht nachgegangen werden. Wir müssen uns hier darauf beschränken, die Titel einiger rechtsgeschichtlich bedeutsamer Formulare nambaft zu machen. Mehr als manches andere beansprucht wohl der S. 21 mitgeteilte ,ordo

1) Andererseits mag man es bedauern, wenn Ludwig Eisenhofer in seiner soeben ausgegebenen und gewiß bedeutenden Neuauflage von Valentin Thalh of $\theta \mathrm{r}$, Handbuch der katholischen Liturgik, Freiburg i. Br. 1912, häufig dort auf Ausblicke auf die kirchliche Rechtsgeschichte verzichtete, wo es der Stoff gebieterisch verlangte. 
benedictionis super sponsum et sponsam" einige Aufmerksamkeit. Dieses Formular schreibt nämlich ausdrücklich vor, der Pfarrer solle die Brautleute über etwa vorhandene Ehehindernisse befragen und solle auferdem deren Willenserklärung fordern, die the zu schließen. Diese Rubrik gibt also zu erkennen, man hat synodale Vorsehriften, die Ehe „in facie ecclesiae" einzugehen, bereits im 13. und 14. Jahrhundert in der Diözese Breslau praktisch - natürlich gab es trotzdem klandestine Ehen - durchgeführt. Wichtiger ist jedoch an der nämlichen Rubrik der weitere Umstand: das Formular gehört zu den frühesten, die neben der Benediktion die Konsenserklärung der Nupturienten "in facie ecclesiae" fordern. Franz ist in keinem älteren in Deutschland geschriebenen Rituale diese Konsenserklärung begegnet. Wir notieren ferner noch die "benedictio anuli“ (S. 21) und für die Bufforschung den "ordo ad imponendam carinam penitentibus" (S. 24). Aber auch die Geschichte der Domkapitel wird gern den nordo ad recipiendos fratres" oder, wie er auch benannt ist ${ }_{n}$ modus et forma recipiendi prelatos et canonicos" (S. 29) vermerken. Franz macht dazu die gewif lehrreiche Bemerkung, diese Gebete seien offenbar aus einem Klosterrituale herübergenommen und dann nicht unpassend für die Aufnahme von Prälaten und Kanonikern in das Gremium des Domkapitels verwendet worden. Wir fügen zu dieser Auslassung eine andere. Es wäre sehr zu begrülien, wenn man einmal dem Problem nachgehen würde: welchen Einfluß hat eigentlich das mittelalterliche Cönobium auf das mittelalterliche Domstift ausgeübt? Waren doch die Klöster die Quellgründe eines fast unerschöpflichen kultgeschichtlich, aber auch verfassungsgeschichtlich denkwürdigen Lebens. Das Domstift hat von diesen sicherlich ungleich mebr empfangen als es ihnen zu geben vermochte. Freilich sind die Vorarbeiten heute wohl noch nicht weit genug gediehen, um diese lockende Fragestellung erschöpfend beantworten zu können. Um von anderm ganz zu geschweigen, sei nur daran erinnert, dafi etwa Adalbert Ebner in seinen Untersuchungen über die klösterlichen Gebetsverbrüderungen (Münchener theol. Diss. 1890) - gaz unzweifelbaft haben gerade die Verbrüderungen in den Beziehungen zwischen Kathedrale und Kloster eine grofe Rolle gespielt - bereits mit der karolingischen Zeit abschliekt.

Die Bearbeiter domstiftischer Geschichte seien schlieflich auch auf das bei Franz mitgeteilte niuramentum prestari solitum per eos, qui in prelatos uel canonicos recipiuntur" (S. 45) verwiesen. Es braucht kaum eigens hervorgehoben werden: die wie angedeutet rechtsgeschichtlich bemerkenswerte Edition des Breslauer Rituale ist auch nach der Seite der Textkritik wie nicht weniger in Hinsicht auf die freigebig gespendeten und wertvollen Erläuterungen eine hervorragende Leistung. Die in Farbendruck beigegebenen sieben Tafeln, die über Schrift, Buchschmuck und Notensätze orientieren, steigern den vorbildlichen Wert dieser Ausgabe.

Wir verbinden mit dieser Anzeige den lebhaften Wunsch, weitere Arbeiten in der Ritualienforschung (über die bisher gedruckten Ritualien 
unterrichtet am besten das bei Franz, Kirchliche Benediktionen I, S. XXX-XXXIV mitgeteilte Verzeichnis) möchten bald nachfolgen. Erst wenn weitere derartige Ausgaben und Untersuchungen vorliegen, wird es dem Liturgiehistoriker, aber auch dem Rechtshistoriker möglich sein, die Bearbeitung eines Problems aufzunehmen, das schon lange einer umfassenden Forschung wartet: wir meinen die Benediktionen der Personen. Es wäre das ein Gegenstück zu Franz' "Kirchliche Benediktionen", in denen ja in der Hauptsache nur die Segnungen über Sachen dargestellt sind, wenn auch über die Benediktionen der Personen vieles und überaus aufschlukreiches hier bereits gesagt wird. Freilich wir zweifeln daran, ob eine einzige Kraft derartige Untersuchungen über die Personen auf sich nehmen wird, wie sie Franz mit starken Schultern für die Sachen geleistet hat. Für ein solches Unternehmen dürfte sich wohl am zweckmäßigsten eine Reihe von Mitarbeitern zusammentun. Freilich, da die Interessen des Publikums für liturgiegeschichtliche Fragen zurzeit noch gering sind, würde eine derartige Serie von Veröffentlichungen sicherlich auch von einer bedeutenden Subvention abhängig sein.

Aber eine energische Aufnahme der Ritualienforschung wäre noch unter einen anderen Gesichtswinkel sehr willkommen. Auch die Rechtshistoriker hätten ein Interesse an der Bearbeitung eines zweiten und wohl noch nirgendwo ernstlicher aufgeworfenen Problems: wie wurde es dem mittelalterlichen Bischof möglich, seine Diözese zu einer liturgischen Einheit umzuformen? Eine solche Untersuchung wäre zweifelsohne ein dankenswerter Beitrag zur Geschichte der Zentralisationsbestrebungen des mittelalterlichen Diözesanbischofs. Gerade in der letzten Zeit haben wir ja manches darüber gehört, welche Wege man fand, um den zerrissenen Diözesankörper einheitlich zusammenzufassen oder überbaupt erst zu gestaltęn. Hierher gehören ebensowohl die Untersuchungen von Hilling über die bischöflichen Offiziale als auch die Ausführungen, die Ulrich Stutz in seinem „Eigenkirchenvermögen" (Festschrift für Otto Gierke, Weimar 1911) zur Entstehungsgeschichte des nepiscopatus“ gemacht hat. Hauck hat uns dazu den Werdegang des bischöflichen Territorialstaates dargelegt. Von anderer Seite wurden wir darauf aufmerksam gemacht, dak der Ordinarius des 12. Jahrhunderts seine Aufsichtsrechte über Klöster und klösterliche Eigenkirchen mit Energie und Erfolg wahrnahm und daf diese seine Politik auch die Billigung der Kurie fand. Ebenso wurde festgestellt, daß die Exemtionen im mittelalterilchen Diözesankörper nicht so verhängnisvoll wirkten, als man bisher gemeiniglich annahm. In den Kreis dieser und ähnlicher Untersuchungen, die die Einheitsbestrebungen der mittelalterlichen Diözese ins Auge fassen, möchten wir auch eine Arbeit über liturgische Unionsversuche und Unionserfolge einbezogen wissen. Die liturgische Entwicklung lief auch hier der rechtsgeschichtlichen parallel. Vielleicht hat hier und da die erste der letzteren sogar die Wege bereitet.

Berlin. Georg Schreiber. 